This is an author produced version of a paper published in Journal of Oral Rehabilitation. This paper has been peer-reviewed but does not include the final publisher proof-corrections or journal pagination.

Citation for the published paper:

Wolf, Eva; Dragicevic, Mille; Fuhrmann, Märta. (2019). Alleviation of acute dental pain from localised apical periodontitis : A prospective randomised study comparing two emergency treatment procedures. Journal of Oral Rehabilitation, vol. 46, issue 2, p. null

URL: https://doi.org/10.1111/joor.12730

Publisher: Wiley

This document has been downloaded from MUEP (https://muep.mah.se) / DIVA (https://mau.diva-portal.org). 
DR. EVA C WOLF (Orcid ID : 0000-0001-7425-1038)

Article type : Original Article

\section{Alleviation of acute dental pain from localized apical periodontitis: a prospective randomized study comparing two emergency treatment procedures}

Running title: Alleviation of symptomatic apical periodontitis

Eva Wolf ${ }^{1}$, Mille Dragicevic ${ }^{1,2}$, Märta Fuhrmann ${ }^{1,3}$

${ }^{1}$ Department of Endodontics, Faculty of Odontology, Malmö University, Malmö, Sweden,

Correspondence : Eva Wolf, Department of Endodontics, Faculty of Odontology, Malmö University, SE-205 06 Malmö, Sweden (Tel.: +46-40-665 85 61; email: eva.wolf@ mau.se)

Acknowledgement

The authors deny any conflicts of interest related to this study.

Footnote

${ }^{2}$ Sundspromenaden 1, 21116 Malmö, Sweden

${ }^{3}$ Public Dental Health Service, Skåne County Council, Malmö, Sweden

\section{Abstract}

Objective

To compare the outcomes of two emergency treatment procedures to alleviate pain from localized symptomatic apical periodontitis: complete chemo-mechanical disinfection (CMD) of the root canal system, or removal of necrotic tissue from the pulp chamber (RNT), i.e. without instrumentation of the root canals.

Methods

Fifty-seven consecutive patients from the emergency clinic at Malmö University met the inclusion criteria: spontaneous pain and/or pain on percussion and palpation, non-bleeding pulp in the canal orifice, pain $\geq 4$ on a Numeric Rating Scale and $\geq 18$ years of age. The diagnosis was symptomatic apical periodontitis, in the absence of swelling and/or fever. Pre-

This article has been accepted for publication and undergone full peer review but has not been through the copyediting, typesetting, pagination and proofreading process, which may lead to differences between this version and the Version of Record. Please cite this article as doi: $10.1111 /$ joor. 12730

This article is protected by copyright. All rights reserved. 
operative pain levels and intake of analgesics were registered. The patients were randomized to one of the two treatment groups. Three to five days post-operatively, the patients were contacted by telephone and asked to grade their current pain level and report any postoperative intake of analgesics and antibiotics.

\section{Results}

Of the patients treated with CMD of the root canal system, 26/30 (87\%) reported satisfactory pain relief, compared with $22 / 27(81 \%)$ of those treated by RNT. There was no mean difference in pain relief between the two groups $(\mathrm{p}=0.879)$. Post-operatively, $37 \%$ in each group reported using analgesics and one in each group reported using antibiotics.

\section{Conclusion}

Three to five days after treatment, a majority $(>80 \%)$ in both groups reported adequate pain relief, in some cases in combination with analgesics. Removal of necrotic and infected tissue from the pulp chamber might therefore be a cost-effective emergency treatment alternative to complete chemo-mechanical disinfection.

Keywords: chemo-mechanical disinfection, necrotic tissue, pain, root canal infection, rubber dam, symptomatic

\section{Background}

In endodontic practice, patients frequently present with acute pain from a necrotic pulp. ${ }^{1}$ Suggested emergency treatments comprise complete chemo-mechanical disinfection of the root canal system and systemic or local antibiotic treatment. This may be a stand-alone procedure, or may be combined with other treatment. ${ }^{2,3,4,5,6}$

In a prospective randomized study, 100\% (10/10) patients presenting with a localized acute apical abscess reported only mild or no pain three days after complete chemo-mechanical disinfection. ${ }^{3}$ In another prospective randomized study of patients with symptomatic necrotic teeth, $91 \%$ (20/22) patients were pain-free or had only mild pain five days after complete chemo-mechanical disinfection. ${ }^{4}$ Systemic antibiotics as the sole treatment can provide pain relief. $^{2}$ However, no additional benefit was reported when antibiotics were used to complement chemo-mechanical disinfection of the root canal system in patients with a symptomatic necrotic tooth. ${ }^{3.4}$ 
Several studies show good results for pulpotomy procedures as emergency treatment in symptomatic vital teeth, with approximately $90 \%$ of patients achieving satisfactory postoperative pain relief. ${ }^{7,8,9}$ This result is comparable with that for complete chemo-mechanical instrumentation of the root canal system in vital teeth. However, as emergency treatment, a pulpotomy would be a more cost-effective option, because of the shorter chairside time required. ${ }^{8,10}$ Another procedure, which may correspond to a pulpotomy as a form of costeffective emergency treatment of necrotic symptomatic teeth, is the removal of the necrotic and infected tissue from the pulp chamber. There is crucial difference between this emergency treatment of a vital and a necrotic, infected tooth. In a vital tooth the infected tissue (dentin) is completely excavated, whereas in the necrotic tooth, although the bulk of the infected tissue is removed, there will be residual necrotic infected tissue in the root canal system. This may have a negative impact on pain relief.

The purpose of emergency treatment for patients suffering from toothache is to reduce the pain to an acceptable level and preferably to remove the causative factors. Patients with acute dental pain present at the clinic without a scheduled appointment. Nevertheless, it is important to provide the emergency treatment as efficiently as possible. Since 1 January 2013, the Swedish general dental insurance scheme has no longer reimbursed complete chemo-mechanical disinfection of the root canal system in necrotic teeth as an emergency treatment: only removal of tissue from the pulp chamber is reimbursed. Thus, this is a routine procedure in general dental practice in Sweden. ${ }^{11,12}$ There is little scientific evidence to support the effectiveness of this procedure. ${ }^{3,4}$

The aim of the present study was to compare the outcomes of two emergency treatment procedures to alleviate the pain from localized symptomatic apical periodontitis: complete chemo-mechanical disinfection of the root canal system (CMD), or removal of necrotic tissue from the pulp chamber (RNT), i.e. without application of a rubber dam and instrumentation of the root canals. It was hypothesized that the CMD emergency treatment procedure would result in pain relief to a higher extent than the RNT.

This article is protected by copyright. All rights reserved. 


\section{Methods}

\section{Subjects}

The participants were drawn from consecutive adult patients presenting at the emergency clinic at the Faculty of Odontology at Malmö University, Malmö, Sweden, from March 2012 until May 2016. The patients were clinically diagnosed with symptomatic apical periodontitis and this diagnosis was proposed in cases of spontaneous pain and/or pain on percussion and palpation in a specified tooth. A diagnosis of necrotic pulp was proposed in cases where there was radiographic evidence of bone destruction and this particular tooth responded negatively to sensitivity tests or excavation. The diagnosis of the tooth included in the study was confirmed by probing the canal orifice/s: the absence of bleeding confirmed the tentative diagnosis, which was made regardless of radiographic evidence of bone destruction. A suspected systemic involvement, manifest as intra- or extra-oral swelling or fever, implied exclusion (Table 1). The patients were included only when an endodontic specialist or resident in endodontics was on duty at the emergency clinic. Seventy patients were assessed for eligibility. Six were excluded: three declined to participate and the other three did not meet the inclusion criteria (Fig. 1).

In all, 64 patients were enrolled. Seven did not complete the study, for the following reasons:

- The pre-determined treatment was not possible, because the root canals were inaccessible (2)

- The pre-determined procedure of cleaning and shaping the canals up to two sizes larger than the first apically binding file was not achieved (1)

- Access opening disclosed a vital pulp (3). One patient was allocated to CMD while two were allocated to RNT.

- The patient had already been enrolled with another tooth (1). This patient was allocated to CMD.

The CMD group comprised 30 patients (11women, 19 men) and the RNT group, 27 patients (14 women, 13 men). The patients were aged between 21 and 88 years, average 46.2 years. Table 2 shows the distribution of tooth type and pre- and postoperative pain levels in each treatment group. 


\section{Treatment}

The examinations and the subsequent emergency treatments were undertaken by undergraduate students under the supervision of a specialist or resident in endodontics, or else by the specialist/resident at the emergency clinic.

Initial registration included the pre-operative pain rating on a Numeric Rating Scale (NRS), a scale from 0-10 where 0 represents "no pain" and 10 "worst pain". Any use of analgesics was also registered: none, non-opioids or opioids. Pre-operative use was organized by the patients themselves and noted after they were questioned about it at their appointment. Non-steroidal anti-inflammatory drugs (NSAID), acetylsalicylic acid (ASA) and paracetamol were registered as non-opioids. A randomization chart created in Microsoft Office Excel 2007 with randomly ordered numbers from 1-1000 was used to allocate the patients to a treatment group: complete chemo-mechanical disinfection of the root canal system (even numbers) or the removal of necrotic tissue from the pulp chamber but without the application of rubber dam and instrumentation of the root canals (odd numbers). The randomization was carried out by the operator immediately after the diagnosis had been made and the patient had agreed to participate.

Any caries was completely excavated and access was prepared in order to expose the orifices of all the root canals. The CMD treatment was undertaken under rubber dam, isolating the working area from the oral fluids. The tooth and clamp were disinfected with a $30 \%$ solution of hydrogen peroxide (Apotek Produktion \& Laboratorier AB, Sweden), while the entire field of operation, including the tooth, clamp, and rubber dam, was disinfected with $0.5 \%$ chlorhexidine alcohol (Fresenius Kabi, Denmark). The root canals were cleaned and shaped using the crown-down concept with K3 rotary instrumentation (Dentsply, DeTrey GmbH, Konstanz, Germany) and/or manual instruments following the ISO standardization, to a size at which necrotic tissue and infected dentin could be adequately removed, i.e. at least ISO file number 35, or two sizes larger than the first apically binding file. The root canals were irrigated with $0.5 \%$ buffered sodium hypochlorite $(\mathrm{NaOCl})$ solution (Dakin's Solution) and $15 \%$ ethylenediaminetetraacetic acid (EDTA) solution (Apotek Produktion \& Laboratorier $\mathrm{AB}$, Sweden). After chemo-mechanical disinfection, all the teeth were dressed with calcium hydroxide and sealed with a temporary dressing of zinc oxide-eugenol cement (ZOE), covered by glass ionomer cement (Ketac ${ }^{\mathrm{TM}}$ Fil, 3M ESPE Dental Products, Germany). 
The RNT treatment was carried out without rubber dam. Necrotic and infected tissue was flushed out of the pulp chamber with non-sterile water. The teeth were then sealed with ZOE and a glass ionomer cement (Ketac ${ }^{\mathrm{TM}}$ Fil, 3M ESPE Dental Products, Germany). A new appointment was scheduled at the Department of Endodontics within two weeks of the emergency treatment, for complete chemo-mechanical disinfection of the tooth under aseptic conditions.

\section{Outcome}

The primary outcome of the present study was adequate pain relief $(\mathrm{NRS}<4)$ after treatment. All the patients were contacted by telephone by one of the authors, using a structured questionnaire, three to five days after the emergency treatment and reminded of their preoperative grading on the NRS. They were then asked to grade their current post-operative pain levels on the same scale; a grading of NRS $<4$ was considered to represent adequate pain relief. ${ }^{13}$ Any post-operative use of analgesics and antibiotics was also registered at this time. There was no blinding. A trial registration was made at ISRCTN 15384527.

\section{Power analysis}

According to the results of Fouad et al. $(1996)^{3}$ and Henry et al. $(2001)^{4}$ adequate pain relief was achieved in (30/32) 94\% of patients undergoing CMD. If an estimated satisfactory pain relief (NRS <4) for $70 \%$ of the patients after an emergency treatment can be considered clinically acceptable, it was calculated that two groups of 29 patients each would be required to achieve a statistically significant result with $80 \%$ power. The power calculation is onesided, based on the assumption that more patients will achieve satisfactory pain relief with CMD than with RNT because in the latter there is residual infection in the root canals.

\section{Statistics}

The Statistical Packages of Social Sciences version 22 (SPSS Inc., Chicago, IL, USA) was used for the calculations. The change in preoperative pain rating within each group was tested with a paired t-test. The statistical method selected was Fisher's exact test of $2 \times 2$ tables, because the proportions were small and the expected value $<5$. The calculations were twotailed, and the independent sample t-test was used for calculations of equality of means in pain relief. Results were considered statistically significant at $\mathrm{p}<0.05$. 


\section{Ethical approval}

The Regional Ethical Review Board at Lund University approved the study (Diary Number 2013/167). The patients received verbal and written information about the study and gave written informed consent at the interview. No compensation was given for participation.

\section{Results}

The average pre-operative pain rating for the patients in the CMD group was NRS 7.0, decreasing to NRS 1.4 postoperatively $(\mathrm{p}<0.001)$. The corresponding NRS ratings in the RNT group were 7.4 and 2.1 respectively $(\mathrm{p}<0.001)$. There was no mean difference in pain relief between the two groups $(\mathrm{p}=0.879)$. In the CMD group, satisfactory pain relief $(\mathrm{NRS}<4)$ was achieved in 26/30 (87\%) patients. The corresponding results for the RNT group were 22/27 $(81 \%)$ patients $(\mathrm{p}=0.722)$. One patient in each group experienced increased pain and local swelling three to five days post-operatively and was prescribed antibiotics. For the distribution of analgesic intake, see Table 3. Post-operative use of analgesics, both opioids and non-opioids, was organized by the patients themselves. Two patients were given prescriptions for antibiotics, one from the first author and the other from a colleague in a foreign country.

\section{Discussion}

For both CMD and RNT, the pain-relieving effect was statistically significant $(\mathrm{p}<0.001)$ and decreased to a level regarded as clinically acceptable (NRS 1.36 and 2.07 respectively), with or without analgesics. Adequate pain relief (NRS <4) was achieved for $87 \%$ and $81 \%$ of the patients, respectively. As both procedures investigated in this study were shown to be satisfactory as an emergency treatment, it might be relevant to try to determine whether the two treatments are equivalent. A substantially larger patient sample would be required for such calculations. ${ }^{14}$

Research on this topic is limited and the outcome is as yet unconfirmed. It was therefore decided to exclude patients with systemic involvement (fever, swelling), in order to minimize any risk of exacerbation of the condition. As RNT does not completely remove infected material, there was a potential risk of further spread of infection. 
Defining a necrotic pulp on the basis of a non-bleeding canal orifice is based on the understanding that pulpal disease progresses gradually. ${ }^{15,16,17}$ As long as the vitality (blood supply) is preserved, there will be no infection in the pulpal space. However, as soon as some part of the pulp becomes necrotic, there is a risk of bacterial invasion and colonization. The presence of non-bleeding necrotic tissue at the canal orifice/s is evidence of partial or total infection of the root canal.

The collected information from the subjects about their experience of post-operative pain was not blinded as to which treatment the subjects had undergone. This might be questioned. However, the potential disadvantage was compensated partly by the use of a structured questionnaire, meaning that the same questions were asked of each subject. However, when necessary, clarifying questions were also occasionally included.

For measuring pain, NRS was chosen instead of the visual analogue scale (VAS) ${ }^{18}$ which has been used previously. ${ }^{3}$ The VAS requires active participation on the part of the patient, and was therefore not considered appropriate for our study, in which patients were asked to grade their postoperative pain over the telephone. Moreover, it is reported that the validity of NRS in detecting differences in the intensity of various painful stimuli is slightly higher than that of the VAS. ${ }^{19}$ Values NRS < 4 correspond to no pain or mild pain. To be able to measure the level of pain relief, patients with a value NRS $<4$ were not included in the present study. ${ }^{20}$ In addition, during telephone contact three to five days post-operatively, we chose to remind the patients of their pre-operative grading because patients tend to overrate their post-operative pain when they do not have access to their previous grading results. ${ }^{21}$

Collecting information on pain status by telephone has of course limitations and precludes objective evaluation of tenderness to pain on percussion or palpation. However, from a clinical perspective (assessing the possible need for additional emergency treatment) the patient's subjective report on pain might be sufficient. ${ }^{22}$

Patients presenting at the emergency clinic with toothache frequently report using analgesics. Some of the participants reported preoperative medication. Their initial NRS score was probably lower than if they had not taken any medication. Any negative impact of this on the results of the current study was however, circumvented, by including only patients who reported NRS $>4$, with or without analgesics. Some patients also reported postoperative 
medication with analgesics. However, the outcome of an emergency treatment must be considered satisfactory from a clinical point of view, when the patient reports adequate pain relief after emergency treatment alone, or in combination with analgesics if necessary. ${ }^{23}$

The lack of a rubber dam application for emergency RNT treatment may be questioned and debated. Application of rubber dam for root canal treatment has several purposes. It is important to protect the field of operation from contamination by the oral flora and to protect the patient from the risk of aspiration of root canal instruments. Rubber dam application also allows disinfection of the operative field: working aseptically avoids contamination by microorganisms other than those already established in infected teeth. No root canal instrumentation is involved in the RNT procedure, hence infected material in the root canals remains undisturbed. Rubber dam application might therefore not be considered necessary at this emergency treatment. For the same reason, that root canal infection is undisturbed, flushing the necrotic infected tissue from the pulp chamber with water could be considered adequate under the conditions of the RNT procedure. Moreover, this procedure is used in general dental practice in Sweden ${ }^{11}$ and the opinion of general dental practitioners is that it functions well. ${ }^{12}$

However, for CMD, rubber dam application, providing a disinfected field of operation, and an aseptic technique, is essential to ensure a good prognosis. ${ }^{24,25}$ In cases of symptomatic apical periodontitis, the preferred procedure is thorough chemo-mechanical disinfection of the root canal system under aseptic conditions and adequate chairside time to allow good quality root canal treatment. However, this study indicates that in an emergency situation, RNT can be considered a cost-effective alternative: most of the patients reported satisfactory pain relief, with or without analgesics, hence very limited need for an additional emergency treatment. Pressure of time might affect the quality of a CMD, increasing the risk of complications and consequently a poorer prognosis. In contrast, emergency RNT can be followed up by a dental appointment allowing adequate time for optimal endodontic treatment, including adequate aseptic procedures, and hence a good prognosis as a sequel. ${ }^{24,25}$

Although it should be interpreted with great caution, a positive finding of the current study was that regardless of treatment, nearly all cases were uncomplicated by postoperative fever and/or swelling, avoiding the need for antibiotics. Since antibiotic resistance development, which is rapidly increasing worldwide and no longer a potential but rather a current threat to 
global public health, ${ }^{26}$ this should be regarded as a promising finding which warrants further study. One issue raised in conjunction with RNT was that leaving infected tissue in the root canals might potentially increase prescription of antibiotics, but this was not confirmed in the present study.

In accordance with the results of this study, the removal of necrotic and infected tissue from the pulp chamber (in combination with analgesics if needed) would be an option for emergency treatment to provide satisfactory pain relief for patients presenting with a necrotic pulp and symptomatic apical periodontitis. However, an appointment for complete chemomechanical disinfection of the root canal system, including the use of rubber dam, should be scheduled already at the emergency appointment. Also important to emphasize is that for patients with fever and/or swelling, the emergency treatment of choice should include complete disinfection of the entire root canal system

\section{Conclusion}

No difference in pain relief could be found three to five days after either complete chemomechanical disinfection of the root canal system or by removal of necrotic tissue from the pulp chamber but without instrumentation of the root canals, in patients suffering from symptomatic apical periodontitis without systemic involvement. In the majority ( $>80 \%)$ of these patients, adequate pain relief seemed to be achieved irrespective of the abovementioned treatments, albeit in some cases in combination with analgesics. As an emergency procedure in general dental practice, removal of necrotic and infected tissue from the pulp chamber to alleviate dental pain from symptomatic apical periodontitis might therefore be a potentially cost-effective treatment alternative to complete chemo-mechanical disinfection.

\section{Source of funding}

This research was supported by the Faculty of Odontology, Malmö University, Malmö, Sweden. 


\section{References}

1. Mansour MH, Cox SC. Patients presenting to the general practitioner with pain of dental origin. Med J Aust 2006;185: 64-67.

2. Adriaenssen CF. Comparison of the efficacy, safety and tolerability of azithromycin and co-amoxiclav in the treatment of acute periapical abscesses. J Int Med Res 1998;26:257-265.

3. Fouad AF, Rivera EM, Walton RE. Penicillin as a supplement in resolving the localized acute apical abscess. Oral Surg Oral Med Oral Pathol Oral Radiol Endod 1996;81:590-595.

4. Henry M, Reader A, Beck M. Effect of penicillin on postoperative endodontic pain and swelling in symptomatic necrotic teeth. J Endod 2001;27:117-123.

5. Segura-Egea JJ, Gould K, Şen BH, Jonasson P, Cotti E, Mazzoni A, Sunay H, Tjäderhane L, Dummer PMH. Antibiotics in Endodontics: a review. Int Endod J 2017;50:1169-1184.

6. Segura-Egea JJ, Gould K, Şen BH, Jonasson P, Cotti E, Mazzoni A, Sunay H, Tjäderhane L, Dummer PMH. European Society of Endodontology position statement: the use of antibiotics in endodontics. Int Endod J 2018;51:20-25.

7. Hasselgren G, Reit C. Emergency pulpotomy: pain relieving effect with and without the use of sedative dressings. J Endod 1989;15: 254-256.

8. Oguntebi BR, DeSchepper EJ, Taylor TS, White CL, Pink FE. Postoperative pain incidence related to the type of emergency treatment of symptomatic pulpitis. Oral Surg Oral Med Oral Pathol 1992;73:479-483.

9. Nyerere JW, Matee MI, Simon EN. Emergency pulpotomy in relieving acute dental pain among Tanzanian patients. BMC Oral Health 2006;6:1.

10. Moskow A, Morse DR, Krasner P, Furst ML. Intracanal use of a corticosteroid solution as an endodontic anodyne. Oral Surg Oral Med Oral Pathol 1984;58: 600604.

11. Koch M, Eriksson HG, Axelsson S, Tegelberg A. Effect of educational intervention on adoption of new endodontic technology by general dental practitioners: a questionnaire survey. Int Endod J. 2009;42:313-21.

12. Koch M, Englander M, Tegelberg A, Wolf E. Successful clinical and organisational change in endodontic practice. A qualitative study. Eur J Dent Educ 2014;18:121-127.

13. Mantha S, Thisted R, Foss J, Ellis JE, Roizen MF. A proposal to use confidence intervals for visual analog scale data for pain measurement to determine clinical significance. Anesth Analg 1993;77:1041-1047.

14. Piaggio G, Elbourne DR, Pocock SJ, Evans SJ, Altman DG; CONSORT Group. Reporting of noninferiority and equivalence randomized trials: extension of the

This article is protected by copyright. All rights reserved. 
CONSORT 2010 statement. JAMA. 2012 Dec 26;308:2594-604.

15. Kakehashi S, Stanley HR, Fitzgerald RJ. The effects of surgical exposures of dental pulps in germfree and conventional laboratory rats. J South Calif Dent Assoc 1966;34:449-451.

16. Moller AJ. Microbiological examination of root canals and periapical tissues of human teeth. Methodological studies. Odontologisk Tidskrift 1966;74 (Suppl):1-380.

17. Nair PN. Light and electron microscopic studies of root canal flora and periapical lesions. J Endod 1987;13:29-39.

18. Huskisson E. Visual analogue scales. In: Melzack R, editor. Pain measurement and assessment, New York: Raven Press, 1983:33-37.

19. Ferreira-Valente MA, Pais-Ribeiro JL, Jensen MP. Validity of four pain intensity rating scales. Pain 2011;152:2399-2404.

20. Dworkin RH, Turk DC, Farrar JT, Haythornthwaite JA, Jensen MP, Katz NP et al. Core outcome measures for chronic pain clinical trials: IMMPACT recommendations. Pain 2005;113:9-19.

21. Scott J, Huskisson EC. Accuracy of subjective measurements made with or without previous scores:an important source of error in serial measurement of subjective states. Ann Rheum Dis 1979;38:558-559.

22. Roland M, Torgersson DJ. What are pragmatic trials? BMJ 1998;31:285.

23. Mhuircheartaigh RJ, Moore RA, McQuay HJ. Analysis of individual patient data from clinical trials: epidural morphine for postoperative pain. Br J Anaesth 2009;103:874881.

24. Peters LB, Wesselink PR. Periapical healing of endodontically treated teeth in one and two visits obturated in the presence or absence of detectable microorganisms. Int Endod J 2002;35:660-667.

25. Weiger R, Rosendahl R, Lost C. Influence of calcium hydroxide intracanal dressings on the prognosis of teeth with endodontically induced periapical lesions. Int Endod J 2000;33:219-226.

26. Antimicrobial resistance, Global report and surveillance WHO; 2014. (http://apps.who.int/iris/bitstream/10665/112642/1/9789241564748_eng.pdf?ua=1) Accessed August 7, 2016

This article is protected by copyright. All rights reserved. 
Table 1 Inclusion and exclusion criteria for patients suffering from symptomatic apical periodontitis without systemic involvement

Inclusion criteria

Exclusion criteria

Spontaneous pain and/or pain on percussion

Previous endodontic treatment

and palpation

Necrotic (non-bleeding) pulp in canal

Horizontal/longitudinal root fracture

orifice/s

Pain $\geq 4$ on NRS $\dagger$

Root resorption

Accepting a phone call within 3-5 days

Marginal bone loss $\geq 1 / 3$

and a new appointment within 2 weeks

$\geq 18$ years of age

Non restorable tooth

Intra- and/or extraoral swelling, fever

Currently on prescribed antibiotics

$†$ NRS - Numeric Rating Scale (0-10 where 0 represents "no pain" and 10 "worst pain"

This article is protected by copyright. All rights reserved. 
Table 2 The distribution of tooth type and pre- and post-operative pain (3-5 days) registered on a Numeric Rating Scale (NRS) $†$ for 57 patients suffering from symptomatic apical periodontitis and subjected to emergency treatment of either complete chemo-mechanical cleaning and disinfection (CMD) or removal of necrotic tissue from the pulp chamber (RNT)

\begin{tabular}{|c|c|c|c|c|}
\hline \multirow[t]{2}{*}{ Treatment group } & \multirow[t]{2}{*}{ Tooth group } & No. of teeth & \multirow{2}{*}{$\begin{array}{l}\text { Preoperative pain } \\
\text { mean }(\mathrm{SD})\end{array}$} & \multirow{2}{*}{$\begin{array}{l}\text { Postoperative pain } \\
\text { mean (SD) }\end{array}$} \\
\hline & & & & \\
\hline \multirow[t]{4}{*}{$\overline{\mathrm{CMD}}$} & molar & 18 & $7.1(1.85)$ & $1.2(1.54)$ \\
\hline & premolar & 10 & $6.6(1.96)$ & $1.6(2.27)$ \\
\hline & incisor & 2 & $8.5(0.71)$ & $2.0(2.82)$ \\
\hline & & 30 & $7.0(1.85)$ & $1.4(1.83)$ \\
\hline \multirow[t]{4}{*}{ RNT } & molar & 20 & 7.5 (1.76) & $1.5(1.76)$ \\
\hline & premolar & 5 & $6.8(1.79)$ & $1.2(2.68)$ \\
\hline & incisor & 2 & $8.5(0.71)$ & $7.5(3.54)$ \\
\hline & & 27 & $7.4(1.72)$ & $1.9(2.55)$ \\
\hline
\end{tabular}

$\lceil$ Numeric Rating Scale 0-10 where 0 reflects no pain and 10 reflects unbearable pain.

This article is protected by copyright. All rights reserved. 
Table 3 Distribution of pre- and post-operative (3-5 days) analgesic medication in 57 patients diagnosed with symptomatic apical periodontitis. Patients were allocated for emergency treatment, either by complete chemo-mechanical cleaning and disinfection (CMD) or by removal of necrotic tissue from the pulp chamber (RNT)

\begin{tabular}{lllllll}
\hline & \multicolumn{2}{l}{ Pre-operative analgesic medication } & \multicolumn{3}{l}{ Post-operative analgesic medication } \\
& None & Non-opioids $\dagger$ & Opioids & None & Non-opioids $\dagger$ & Opioids \\
CMD & 6 & 17 & 7 & 19 & 8 & 3 \\
RNT & 6 & 17 & 4 & 17 & 8 & 2 \\
\hline
\end{tabular}

No statistically significant difference between the groups was found.

$\dagger$ non-steroidal anti-inflammatory drugs (NSAID), acetylsalicylic acid (ASA), paracetamol 


\section{Figure Legends}

Figure 1.

Flow Diagram on eligibility, exclusion, inclusion and drop-outs for 70 patients with symptomatic apical periodontitis

Figure 1

\section{Flow Diagram}

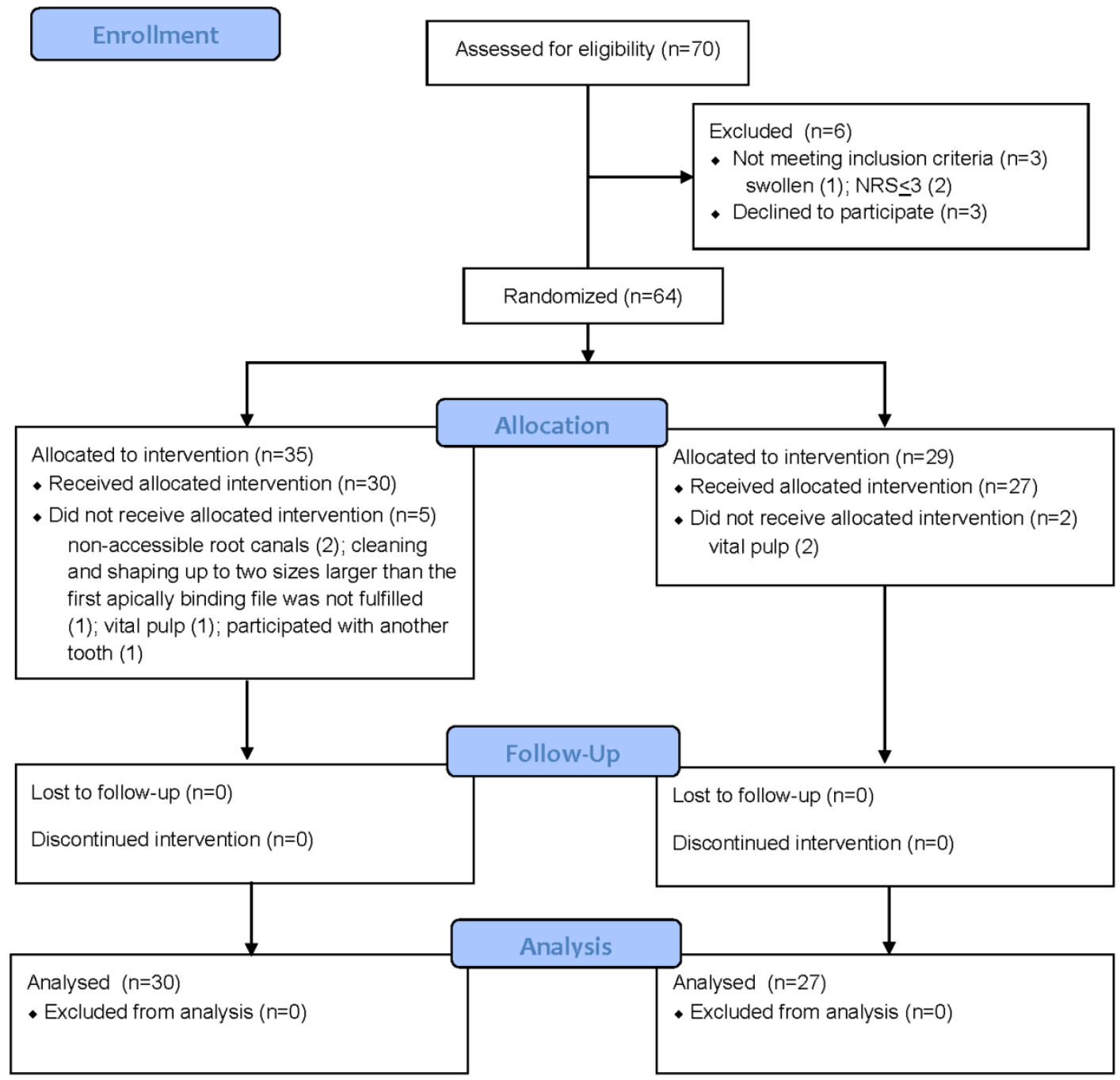

This article is protected by copyright. All rights reserved. 